\title{
Recurrent Spontaneous Pneumothorax During Pregnancy; A Case Report
}

\author{
Andrew J. Weaver, MD', Wai Hau, MS4 ${ }^{1}$, Mark Cooper, MD, PhD'
}

\section{ABSTRACT}

Spontaneous pneumothorax is a rare but potentially life-threatening condition during pregnancy. We discuss a case of recurrent spontaneous pneumothorax during a single pregnancy. The pneumothorax occurred twice during the pregnancy in the second and third trimesters. Both pneumothoraxes were managed conservatively as due to the low prevalence of this condition, management guidelines are few. We report our management of recurrent spontaneous pneumothorax during pregnancy to support the literature and current guidelines.

\section{KEYWORDS}

Spontaneous pneumothorax, pregnancy
Author affiliations listed at the end of this article.

Correspondence to:

Andrew J. Weaver Marshall University Joan C. Edwards School of Medicine weaveran@marshall.edu

\section{INTRODUCTION}

Spontaneous pneumothorax is rare during pregnancy, with only 56 cases having been reported in literature. ${ }^{1}$ Pneumothorax is a potentially life-threatening condition to both the mother and the fetus due to physiological changes not being well-tolerated in pregnancy. Of the reported cases, it appears that approximately 10 percent of pregnant females with spontaneous pneumothorax have a positive family history. ${ }^{2}$ Other known common risk factors include respiratory infections, asthma, and/or a personal history of pneumothorax. ${ }^{3}$ Here, we present a case of a patient with recurrent spontaneous pneumothorax during a pregnancy without any identifiable risk factors.

\section{CASE REPORT}

A 26-year-old gravida 2 para 1 female presented to the emergency department 24.1 weeks pregnant with complaints of a four-hour history of pleuritic chest pains that awakened her from sleep. She denied ever having symptoms such as these in the past. This pain was sudden in onset and worsened with deep inspiration. She reported mild shortness of breath but denied having any recent cough/congestion, fevers, or recent trauma. Her physical exam was unremarkable, and an EKG showed normal sinus rhythm and negative troponins. She underwent a CT scan of her chest to rule out pulmonary embolism and was found to have approximately 10 percent left-sided pneumothorax (Figure 1). She was placed on high-flow nasal cannula, and oxygen saturations were 100 percent. Of note, her past medical history was not significant for

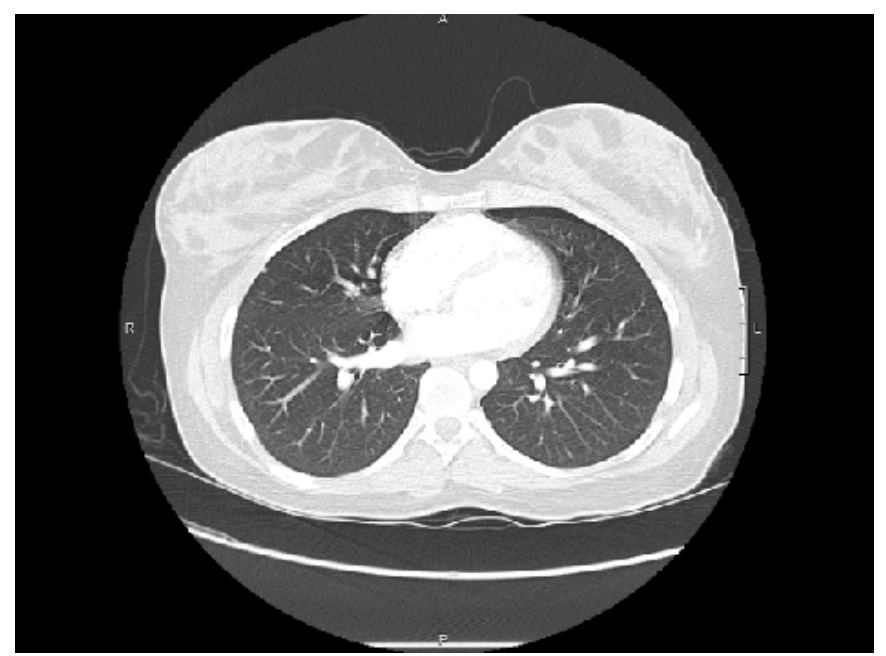

Figure 1: Axial CT scan of chest with IV contrast at the time of first admission showing small left pneumothorax. 


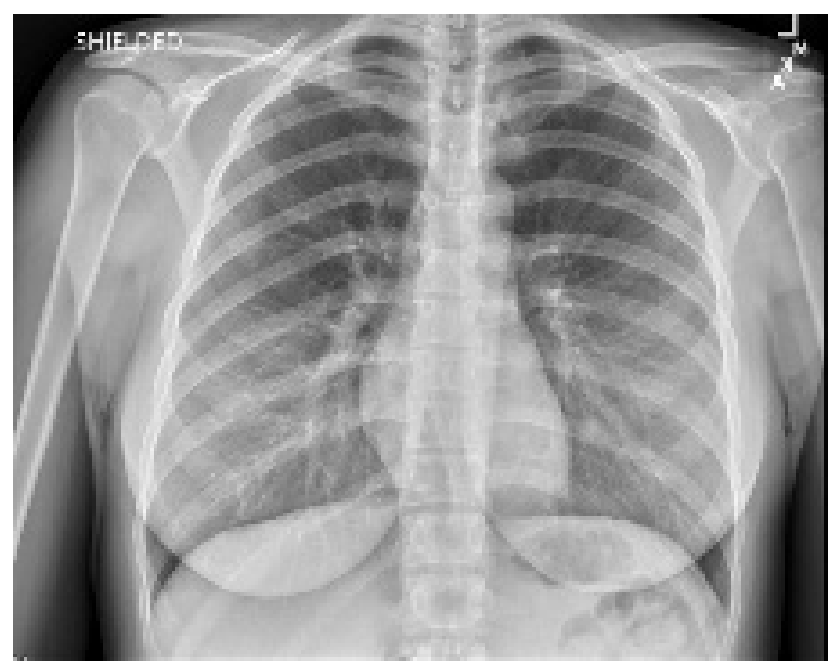

Figure 2a: AP Chest X-ray without demonstrable pneumothorax on hospital day one.

any pulmonary pathology such as infections, asthma, or previous pneumothorax, and she was an otherwise healthy non-smoker. Her only past surgical history was a cesarean due to non-reassuring fetal heart tones during her last pregnancy.

Her pain improved overnight, and the following morning she underwent a two-view chest x-ray (Figures $2 a / 2 b$ ) which did not demonstrate an appreciable pneumothorax. Her high-flow nasal cannula was weaned, and she maintained good oxygenation saturation on room air and reported complete resolution of symptoms. She was discharged without further incident.

Two months later, the patient presented a second time to the emergency department, now 32.5 weeks pregnant, with complaints similar to those of her previous visit. She awoke from sleep with sharp chest pains and mild shortness of breath. She denied any recent cough/congestions or trauma. She underwent an initial chest X-ray with no pathological findings. She then underwent a CT scan of her chest (Figure 3), which again demonstrated a small left pneumothorax. She was admitted to the hospital and placed on high-flow oxygen. She did not have any episodes of oxygen desaturations. The following morning, a chest X-ray did not note any pneumothorax (Figure 4). Her nasal cannula was weaned, and she reported resolution of symptoms. She was later discharged home without further incident. Of note, no blebs or air space disease were reported on chest CT scans.

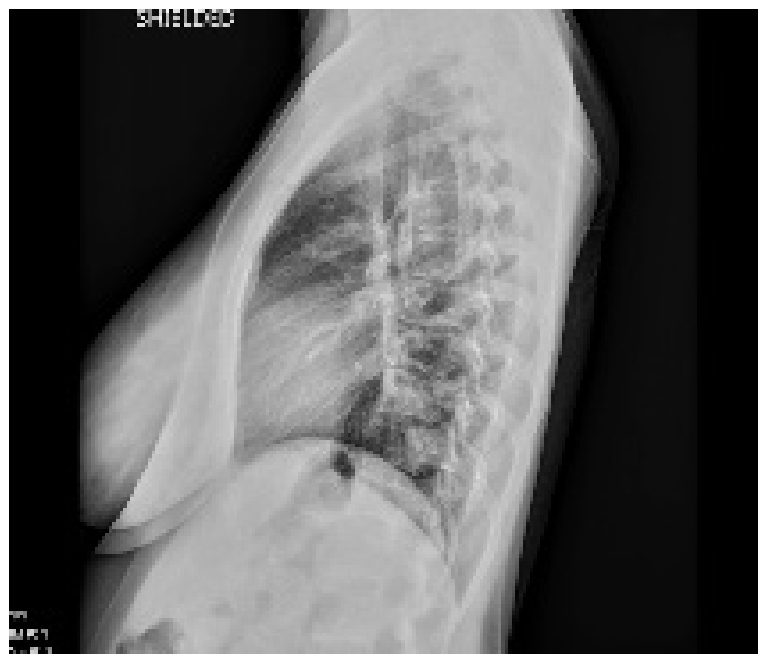

Figure $2 \mathrm{~b}$ : Lateral Chest X-Ray without demonstrable pneumothorax on hospital day one.

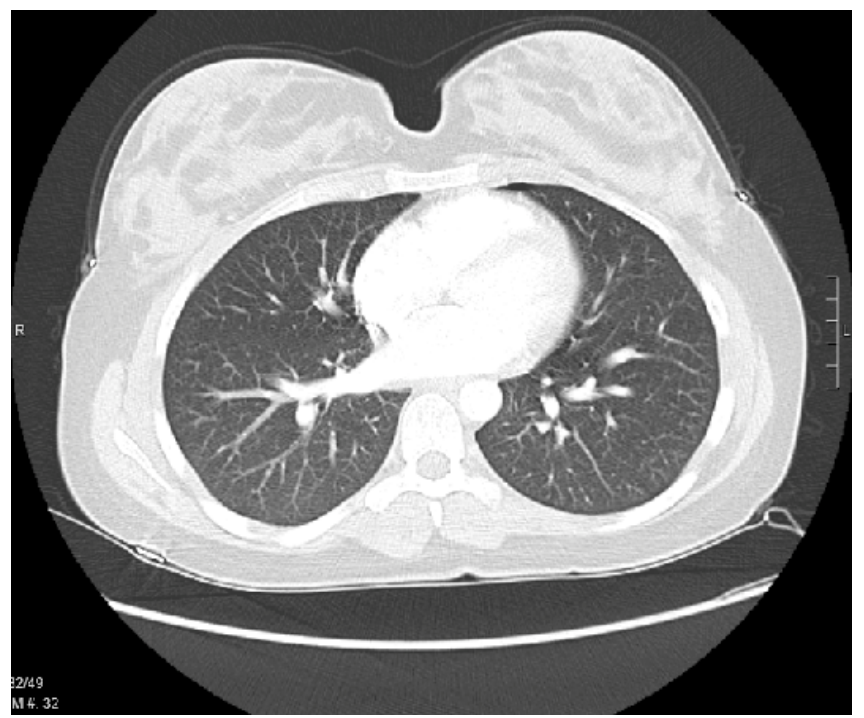

Figure 3: Axial CT scan of chest with IV contrast at the time of second admission with small left pneumotho$\operatorname{rax}$

Eleven months after her previous scan, she had a repeat chest CT scan and it showed no pneumothorax, no blebs, and no airspace disease.

\section{DISCUSSION}

The exact incidence of spontaneous pneumothorax in pregnancy is not known, but it is considered to be a rare event. Although uncommon, it can have significant consequences, including mortality to both the patient and the fetus. Impaired ventilation is not as well tolerated during pregnancy due to a decrease in 


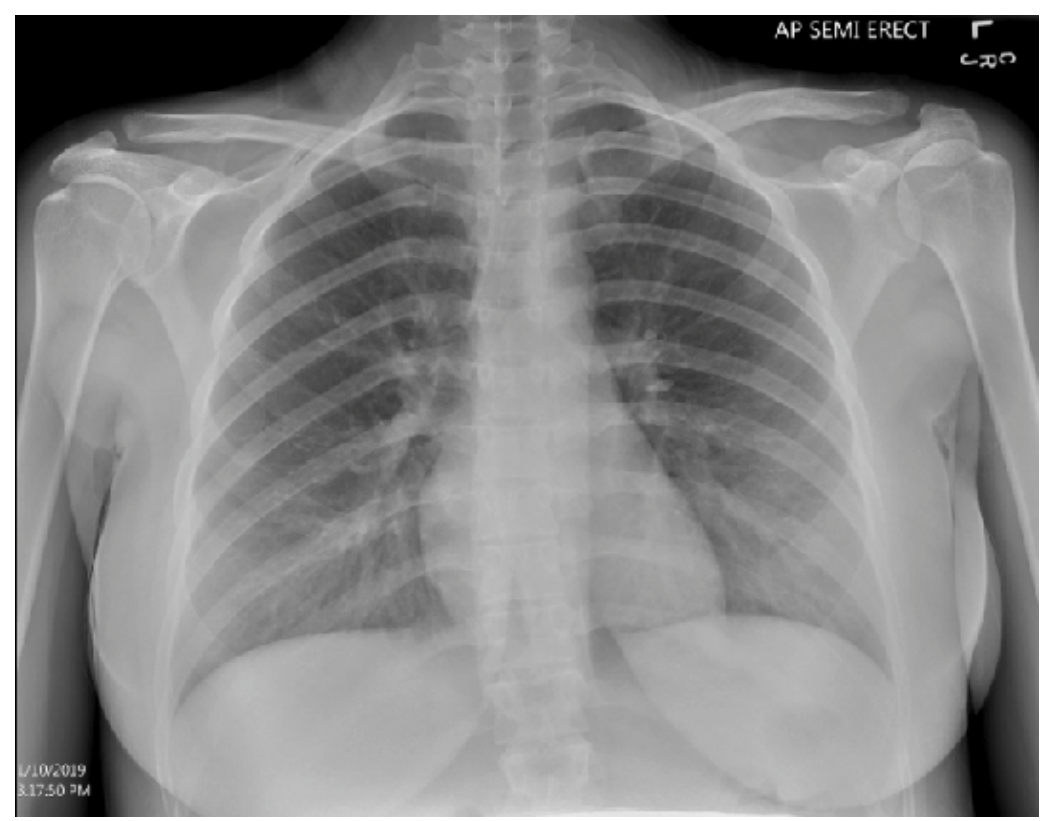

Figure 4: Chest X-ray at second admission without findings of pneumothorax on hospital day one.

functional residual capacity as well as increased oxygen demand of approximately $20 \%$ during pregnancy and $50 \%$ during labor. ${ }^{4}$

Common risk factors include asthma, respiratory infection, and a personal or familial history of spontaneous pneumothorax. Less common risk factors that have been reported include hyperemesis, cocaine use, lymphangioleiomyomatosis, Marfan's, and alpha-one antitrypsin deficiency. ${ }^{5-8}$ Another risk factor for spontaneous pneumothorax is obstructive sleep apnea, which has been shown to be increased in pregnancy. ${ }^{9,10}$ The risk of pneumothorax appears unevenly distributed during pregnancy with 51 percent occurring during the first two trimesters and 49 percent in the third trimester and the perinatal period. ${ }^{3}$ Our patient did not have any of the risk factors mentioned above. The risk of recurrent pneumothorax, as seen in our case, is approximately 30 percent. ${ }^{11}$

The presenting symptoms are typically pleuritic chest pain with dyspnea. Findings on physical examination can vary from normal to absent breath sounds, desaturations, and unstable vitals, depending on the degree of pneumothorax. The diagnosis in pregnancy is made the same as the non-obstetric patient with chest X-ray and CT chest if needed. Chest X-ray has poor sensitivity in detecting pneumothorax, and thus
CT scans are often used. Ultrasonography may be of some benefit as an alternative imaging modality but is very user dependent. It is important to note that chest X-ray and CT scans of the chest do not approach the accepted safe cumulative dose of ionizing radiation during pregnancy and thus should be utilized readily in the diagnosis of obstetric patients.

There is not a developed treatment algorithm for pneumothorax during pregnancy. From a review of the literature, it appears that for a small pneumothorax of less than 15 to 20 percent, the patient should be admitted to the hospital for close observation. A high-flow nasal cannula should be considered, as well as follow-up imaging to ensure resolution of pneumothorax. For a more significant pneumothorax of greater than 15 to 20 percent, simple aspiration and/or thoracostomy tube may be utilized safely. ${ }^{12}$ For patients such as ours with recurrent pneumothorax, thoracotomy or a VATS procedure may be considered. In our case, as the pneumothorax was small and the patient demonstrated resolution with observation, non-operative management with close observation was followed. If the patient is late in her pregnancy, a temporizing thoracostomy tube may be considered and definitive surgery delayed until post-partum. ${ }^{13}$ The safest time for an operative intervention during pregnancy is during the second trimester. Thorascopic approaches have been noted in literature during pregnancy with the benefits of decreased time under an anesthetic, rapid expansion of lung, and decreased post-operative pain.

Regardless of if the patient treatment involved observation, thoracostomy tube placement, or operative intervention, none of these treatments are an absolute indication for cesarean birth. The method of birth should be left to obstetrical indications. This highlights the importance of a multi-disciplinary approach between thoracic surgery, obstetrics, pulmonology, and anesthesia. 


\section{CONCLUSION}

In conclusion, spontaneous pneumothorax is a rare phenomenon during pregnancy, but one that requires fastidious diagnosis and treatment. The complications from a delay in either diagnosis or treatment may yield increased morbidity and possibly mortality of the mother and/or the fetus. Further cases will aid guidelines for the diagnosis and treatment of this condition.

\section{AUTHOR AFFILIATIONS}

1. Marshall University Joan C. Edwards School of Medicine, Department of Surgery

\section{REFERENCES}

1. Lateef N, Dawood M, Sharma K, Tauseef A, Munir MA, Godbout E. Recurrent spontaneous pneumothorax in pregnancy - a case report and review of literature. J Community Hosp Intern Med Perspect. 2018;8(3):115-8.

2. Liberis A, Tsikouras P, Liberis V. Recurrent pneumothorax in a pregnant woman with a family history of spontaneous pneumothorax. Hippokratia. 2015;19(3):287.

3. Garg R, Sanjay, Das V, Usman K, Rungta S, Prasad R. Spontaneous pneumothorax: an unusual complication of pregnancy--a case report and review of literature. Ann Thorac Med. 2008;3(3):104-5.

4. Kavurmaci O, Akcam TI, Kavurmaci SA, Turhan K, Cagirici U. Pneumothorax: A Rare Entity During Pregnancy. Turk Thorac J. 2019;20(3):206-8.

5. Nakamura H, Konishiike J, Sugamura A, Takeno Y. Epidemiology of spontaneous pneumothorax in women. Chest. 1986;89(3):378-82.

6. Warren SE, Lee D, Martin V, Messink W. Pulmonary lymphangiomyomatosis causing bilateral pneumothorax during pregnancy. Ann Thorac Surg. 1993;55(4):998-1000.

7. Chan L, Pham H, Reece EA. Pneumothorax in pregnancy associated with cocaine use. Am J Perinatol. 1997;14(7):385-8.

8. Morrison PJ, Lowry RC, Nevin NC. Familial primary spontaneous pneumothorax consistent with true autosomal dominant inheritance. Thorax. 1998;53(2):151-2.
9. Dominguez JE, Street L, Louis J. Management of Obstructive Sleep Apnea in Pregnancy. Obstet Gynecol Clin North Am. 2018;45(2):233-47.

10. Tulek B, Kanat F, Yosunkaya S, Ceran S, Suerdem $M$. Pneumothorax as an initial manifestation of obstructive sleep apnea syndrome. Sleep Breath. 2010;14(3):249-51.

11. Lal A, Anderson G, Cowen M, Lindow S, Arnold AG. Pneumothorax and pregnancy. Chest. 2007;132(3):1044-8.

12. VanWinter JT, Nichols FC, 3rd, Pairolero PC, Ney JA, Ogburn PL, Jr. Management of spontaneous pneumothorax during pregnancy: case report and review of the literature. Mayo Clin Proc. 1996;71(3):249-52.

13. Jain P, Goswami K. Recurrent spontaneous pneumothorax during pregnancy: a case report. J Med Case Rep. 2009;3:81. 